\title{
Association between ORMDL3, ILIRL1 and a deletion on chromosome 17q21 with asthma risk in Australia
}

\author{
Manuel AR Ferreira ${ }^{\star, 1}$, Allan F McRae ${ }^{1}$, Sarah E Medland ${ }^{1}$, Dale R Nyholt ${ }^{1}$, Scott D Gordon ${ }^{1}$, \\ Margaret J Wright ${ }^{1}$, Anjali K Henders ${ }^{1}$, Pamela A Madden ${ }^{2}$, Peter M Visscher ${ }^{1}$, Naomi R Wray ${ }^{1}$, \\ Andrew C Heath ${ }^{2}$, Grant W Montgomery ${ }^{1}$, David L Duffy ${ }^{1}$ and Nicholas G Martin ${ }^{1}$
}

\begin{abstract}
Genome-wide association studies followed by replication provide a powerful approach to map genetic risk factors for asthma. We sought to search for new variants associated with asthma and attempt to replicate the association with four loci reported previously (ORMDL3, PDE4D, DENND1B and IL1RL1). Genome-wide association analyses of individual single nucleotide polymorphisms (SNPs), rare copy number variants (CNVs) and overall CNV burden were carried out in 986 asthma cases and 1846 asthma-free controls from Australia. The most-associated locus in the SNP analysis was ORMDL3 (rs6503525, $P=4.8 \times 10^{-7}$ ). Five other loci were associated with $P<10^{-5}$, most notably the chemokine CXC motif ligand $14(C X C L 14)$ gene (rs31263, $P=7.8 \times 10^{-6}$ ). We found no evidence for association with the specific risk variants reported recently for $P D E 4 D$, DENND1B and ILR1L1. However, a variant in IL1RL1 that is in low linkage disequilibrium with that reported previously was associated with asthma risk after accounting for all variants tested (rs10197862, gene wide $P=0.01$ ). This association replicated convincingly in an independent cohort $\left(P=2.4 \times 10^{-4}\right)$. A 300-kb deletion on chromosome $17 \mathrm{q} 21$ was associated with asthma risk, but this did not reach experiment-wide significance. Asthma cases and controls had comparable CNV rates, length and number of genes affected by deletions or duplications. In conclusion, we confirm the association between asthma risk and variants in ORMDL3 and identify a novel risk variant in IL1RL1. Follow-up of the 17q21 deletion in larger cohorts is warranted. European Journal of Human Genetics (2011) 19, 458-464; doi:10.1038/ejhg.2010.191; published online 8 December 2010
\end{abstract}

Keywords: whole-genome; gene; atopy; heterogeneity; structural; IKZF3

\section{INTRODUCTION}

Asthma currently affects $10 \%$ of the Australian population and is responsible for 1 million work days lost, 36000 hospital admissions, 402 deaths and $\$ 606$ million a year in direct costs. ${ }^{1}$ A complete understanding of the genetic and environmental risk factors for asthma is critical to design new treatments or prevention strategies that can reduce the disease burden. This is a challenging task, as asthma is a complex disease that involves the interplay between multiple physiological processes. $^{2}$

Genome-wide association studies (GWAS) currently provide the most powerful approach to identify genetic risk factors for complex diseases. In the past few years, hundreds of variants with replicated associations have been identified for a range of complex diseases or traits using this approach. ${ }^{3,4}$ As hoped, several of these discoveries have identified aetiologic pathways not previously implicated in these traits, such as the autophagy pathway in Crohn's disease ${ }^{5}$ and the HLA-C locus in control of viral load in HIV infection. ${ }^{6}$

Seven GWAS of asthma have been published recently. ${ }^{7-13}$ From these, three loci have emerged with reproducible effects on asthma risk in independent samples: the GSDMB/ORMDL3 locus (henceforth referred to as ORMDL3) on chromosome $17 \mathrm{q} 12-21 ;^{7}$ the PDE4D gene on chromosome $5 \mathrm{q} 12^{9}$ and the DENND1B on chromosome 1q31. ${ }^{11}$ Three GWAS of asthma biomarkers were also published recently. ${ }^{14-16}$ Quantitative trait loci were identified for the respective phenotype analysed in each study, but for only one of these there was also a convincing association with asthma, the IL1RL1 gene on chromosome 2q12. ${ }^{16}$ A variant at this locus was a significant predictor of eosinophil levels while also reproducibly increasing asthma risk.

Although single nucleotide polymorphisms (SNPs) are now routinely tested for association in GWAS for many common diseases, structural variants, a widespread class of genetic polymorphisms that range in size from only a few base pairs to whole chromosomal rearrangements, ${ }^{17}$ remain much less well studied. The Welcome Trust Case Control Consortium recently performed a GWAS of common copy number variants (CNVs) for eight common diseases, but failed to identify any new risk loci that had not been previously reported through the analysis of SNPs. ${ }^{18}$ These results suggest that common $\mathrm{CNV}$ are unlikely to contribute greatly to the genetic basis of common diseases, but leave open the possibility that rarer structural variants may have a greater impact on risk, as shown for obesity, ${ }^{19}$ autism $^{20}$ and schizophrenia. ${ }^{21}$ The only published genome-wide association analysis between $\mathrm{CNV}$ data and asthma identified a region on chromosome $7 \mathrm{p} 14$ that appeared to be associated with asthma risk, although closer inspection of the region could not rule out the possibility that this was a false-positive association as a result of a biological artefact. ${ }^{22}$

Therefore, the identified variants in ORMDL3, PDE4D, DENND1B and ILIRL1 arguably represent the most convincing associations between genetic variants and asthma risk reported to date. In this

\footnotetext{
${ }^{1}$ Queensland Institute of Medical Research, Brisbane, QLD, Australia; ${ }^{2}$ Washington University School of Medicine, St Louis, MO, USA

*Correspondence: Dr MAR Ferreira, Genetic Epidemiology Laboratory, Queensland Institute of Medical Research, P.O. Royal Brisbane Hospital, Brisbane, QLD 4029, Australia. Tel: +61 73845 3552; Fax: +61 73362 0101; E-mail: manuel.ferreira@qimr.edu.au
}

Received 10 August 2010; revised 28 September 2010; accepted 5 October 2010; published online 8 December 2010 
study, we analysed whole-genome genotype data from 986 asthma cases and 1846 disease-free controls of European descent from the Australian population to (1) search for new sequence or structural variants with strong effects on asthma and (2) confirm the previously reported associations with ORMDL3, PDE4D, DENND1B and IL1RL1.

\section{MATERIALS AND METHODS}

\section{Case and control samples}

The samples analysed herein are a subset of a larger cohort of 16140 individuals ascertained in several waves from the Australian general population and genotyped recently as described in detail elsewhere. ${ }^{23}$ Of these, we selected for analysis 986 unrelated cases and 1846 controls based on the presence (cases) or absence (controls) of self-reported lifetime asthma, as reported in at least one lifestyle or health questionnaire completed as part of six epidemiological studies conducted at the Queensland Institute of Medical Research (QIMR) (Supplementary Table 1). Using SOLAR, ${ }^{24}$ we estimate that this phenotype is $61 \%$ heritable, based on a polygenic model fitted to 2054 individuals for whom phenotype information was also available for 3154 of their relatives (parents and/or siblings)

\section{Whole-genome genotyping and imputation of HapMap SNPs}

Genotype data were obtained with Illumina $317 \mathrm{~K}, 370 \mathrm{~K}$ or $610 \mathrm{~K}$ arrays and stringent quality control $(\mathrm{QC})$ procedures applied to ensure high data quality as described in detail previously ${ }^{23}$ and summarised in Supplementary Table 2. After QC, genotype data were available for 16140 individuals and 561815 SNPs. To increase whole-genome coverage, we inferred unmeasured HapMap SNPs using MACH (http://www.sph.umich.edu/csg/abecasis/MACH/) and the phased haplotype data from the CEU HapMap samples (phase I+II, release22, build 36). Following imputation, we restricted our analysis to 2.38 million SNPs that were genotyped or imputed with high confidence $\left(r^{2}>0.3\right)$ and had an MAF $>0.01$, Hardy-Weinberg equilibrium test $P$-value $>10^{-6}$ in controls and $<5 \%$ missing data in the selected sample of 2832 individuals with asthma information. Individuals were confirmed as unrelated through pair wise wholegenome identity-by-descent analysis.

\section{Population substructure and whole-genome SNP association analyses}

All analyses were performed with PLINK. ${ }^{25}$ To address whether subtle population substructure could have an impact on the association results, we performed a multidimensional scaling (MDS) analysis of identity-by-state (IBS) distances calculated between all pairs of individuals using a subset of SNPs in linkage equilibrium. For reference, we included the 11 HapMap III populations. ${ }^{26}$ To test whether cases and controls were well matched with respect to ancestry, we applied a permutation test for between-group IBS differences. A significant test would imply that cases and controls are unlikely to belong to the same population, which could lead to spurious association results. The main association analysis was performed using a standard allelic test. Age-of-onset information was only available for $20 \%$ of cases, and so was not considered for analysis. Lifetime smoking status, which was available for $96 \%$ of individuals (38\% ever smokers), was not a significant predictor of asthma in this sample $(P=0.82)$ and so was not included as a covariate.

\section{Whole-genome $\mathrm{CNV}$ analysis}

CNV segments were identified for a subset of 759 individuals that participated in the longitudinal Brisbane Adolescent Twin Study (270 asthma cases and 489 controls; Supplementary Table 1) using the program QuantiSNP v1.127 based on the SNP and CNV probes present in the Illumina $610 \mathrm{~K}$ arrays. The default program settings were used in addition to a maximum copy number of four and GC correction. To minimise false-positive CNV calling, we restricted our analysis to large $(>100 \mathrm{~kb}$ and $<1 \mathrm{Mb})$ deletions or duplications called with high confidence (log Bayes' factor $>10$ and with $>10$ probes). We also dropped common CNVs (MAF > 0.05 ), as these were likely well tagged by SNPs.

We performed two sets of association analyses between CNVs and asthma risk. First, we investigated whether the frequency of deletions or duplications at specific loci was associated with asthma status. This is equivalent to the genome-wide SNP analysis, except that a small number of rare structural variants are tested for association instead of a large number of common SNPs. Significance was estimated through the analysis of 10000 permutations.

Second, we tested the hypothesis that the presence of multiple deletions or duplications across an individual's genome (ie CNV burden) may significantly increase the risk of developing asthma. Specifically, we addressed the following questions: (A) do asthma cases have on average more CNVs than controls? In those individuals having at least one $\mathrm{CNV}$, do cases have on average (B) larger CNVs than controls or (C) more genes affected by CNVs than controls? For (A) and (B), the test applied was a logistic regression of disease status on the number of observed CNVs and CNV length (mean or total), respectively, while accounting for potential Illumina batch and plate effects. To determine whether more genes were affected by CNVs in cases than controls (C), we downloaded the genomic coordinates for 18212 genes from the Mar 2006 UCSC browser (based on the longest isoform $\pm 50 \mathrm{~kb}$ ) and determined for each individual how many genes in total were intersected by a CNV (gene count). The test was then a logistic regression of disease status on gene count, while accounting for an individual's number of CNVs, mean CNV length, batch and plate effects. Significance was estimated from 10000 permutations.

\section{Follow-up of top SNP and CNV associations}

We attempted to replicate the top SNP and CNV associations in an independent cohort of 604 individuals, including 391 doctor-diagnosed asthmatics (Supplementary Table 3). These individuals were ascertained from the same QIMR studies that contributed data to the GWAS (Supplementary Table 2) and were recently typed with Illumina $610 \mathrm{~K}$ arrays as part of an additional, as yet unpublished wave of genotyping conducted in the first semester of 2010. All samples are of European descent and passed the standard QC filters described above for the GWAS. Data from this wave were available at this stage only for replication of specific associations.

\section{RESULTS}

\section{Population substructure analyses}

We first performed an MDS analysis of IBS distances to identify subtle ancestry differences between samples that could potentially have an impact on the association results. As expected, most of the 2832 Australian samples analysed clustered closely with the CEU HapMap reference panel, indicating that most individuals have a homogeneous European ancestry (Supplementary Figure 1A). However, two groups of individuals clustered close to, but just outside this larger group of homogeneous European ancestry: one group clustered along the European-African ancestry axis, while the second group clustered along the European-Asian axis. The first group likely represents samples with a southern European ancestry (eg Maltese Australians), as indicated by the partial overlap with the HapMap samples from Tuscany in Italy (TSI) (Supplementary Figure 1B). The second group consists of samples with a predominant European ancestry that is partially mixed with Asian ancestry, as reflected by the small overlap with the HapMap Mexican ancestry samples from Los Angeles (MEX). Importantly, however, this subtle population substructure was found to be independent of case-control disease status ( $P=0.775$; Supplementary Figure $1 \mathrm{C}$ ), suggesting that it would not significantly bias whole-genome association results. Consistent with this observation, the genomic inflation factor for the main association analysis was 1.006 (Supplementary Figure 1D), thus confirming that population substructure or other technical artefacts had a minimal impact on the results.

\section{Whole-genome SNP association results}

Given that the 986 asthma cases and 1846 asthma-free controls were well matched with respect to ancestry, we then applied a standard allelic test of association between individual SNPs and lifetime disease 
Table 1 Regions most associated with asthma $\left(P<10^{-5}\right)$ in the genome-wide SNP analysis

\begin{tabular}{|c|c|c|c|c|c|c|}
\hline \multirow[b]{2}{*}{ Chromosome, bp position } & \multirow[b]{2}{*}{$S N P$, reference allele ${ }^{a}$} & \multicolumn{5}{|c|}{ Allele frequency } \\
\hline & & Closest gene, distance $k b^{b}$ & Cases & Controls & Odds ratio & P-value \\
\hline 17,35348700 & rs6503525, C & ORMDL3, 11 & 0.50 & 0.43 & 1.33 & $4.8 \times 10^{-7}$ \\
\hline 7, 38792860 & rs17700752, G & VPS41\#, 62 & 0.15 & 0.20 & 0.71 & $4.4 \times 10^{-6}$ \\
\hline 22,34352607 & rs4622835, C & LOC284912, 378 & 0.53 & 0.46 & 1.29 & $4.6 \times 10^{-6}$ \\
\hline 10,130181728 & rs7893667, A & MKI67, 367 & 0.35 & 0.29 & 1.31 & $6.1 \times 10^{-6}$ \\
\hline
\end{tabular}

aThe SNPs listed were imputed with high confidence using MACH. With the exception of rs7893667, nearby genotyped SNPs provided comparable evidence for association (illustrated in Figure 1a for the $17 q 12$ region).

bDistance to first base pair of the first exon or last base pair of the last exon, whichever is smallest. A pound (\#) indicates that the SNP is located within the gene.
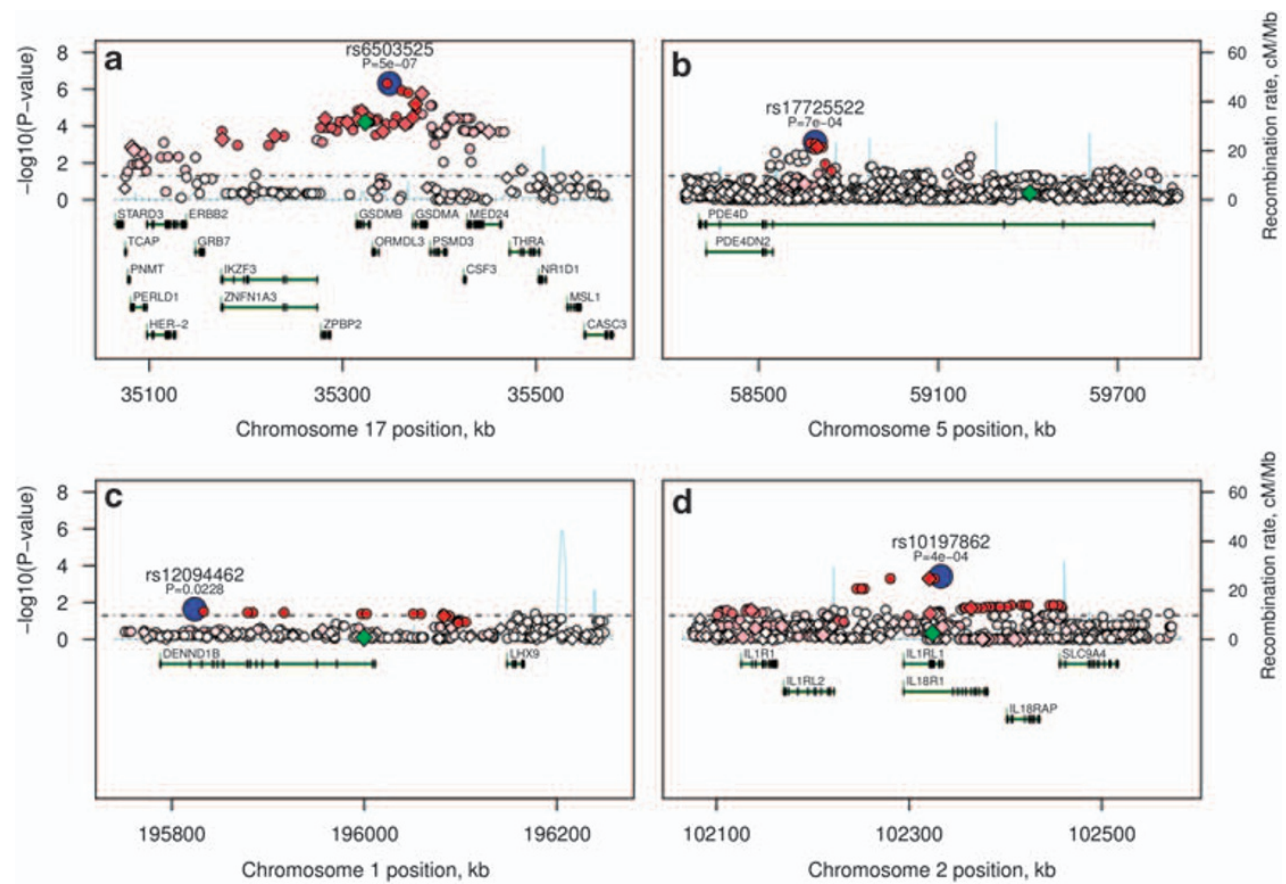

Figure 1 Association plots for the four loci previously reported to associate with asthma risk (a-d). The most-associated SNP for each region is shown in blue, and the colour of the remaining markers reflects the linkage disequilibrium $\left(r^{2}\right)$ with the top SNP in each panel (increasing red hue associated with increasing $r^{2}$ ). The most-associated SNP reported in the original study for each gene is shown in green. Imputed SNPs are represented by circles and genotyped SNPs by diamonds. The recombination rate (second $y$ axis) is plotted in light blue and is based on the CEU HapMap population. Exons for each gene are represented by vertical bars.

status to search for new susceptibility loci for asthma. We acknowledge that given the modest sample size, power was only adequate $(80 \%)$ at the genome-wide level $\left(\alpha=5 \times 10^{-8}\right)$ to detect loci with strong effects (eg an OR $>1.4$, for an allele frequency of $40 \%$ ). The loci most associated with asthma risk $\left(P<10^{-5}\right)$ in this analysis are listed in Table 1, with the full genome-wide results displayed in Supplementary Figure 2. No variant exceeded the widely used threshold for genomewide significance of $5 \times 10^{-8}$, but the most-associated SNP was located

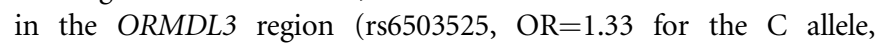
$P=4.8 \times 10^{-7}$ ), a locus previously reported to have strong and reproducible effects on doctor-diagnosed asthma. ${ }^{7}$ Multiple variants in this region were associated with disease risk in our analysis (Figure 1a). The rs6503525 SNP is in linkage disequilibrium (LD, $\left.r^{2}=0.61\right)$ with the top ORMDL3 variant (rs7216389) reported in the original study by Moffat et al; ${ }^{7}$ both predisposing alleles (rs6503525:C and
rs7216389:T) are in phase. We found no evidence for additional independent risk variants in this region (not shown), consistent with Moffatt et al. ${ }^{7}$ These results thus confirm that a genetic variant in the ORMDL3 locus significantly influences asthma risk in the Australian population.

Among the five remaining regions most associated with asthma (Table 1), only for chromosome $5 \mathrm{q} 31$ there was an obvious candidate gene under the association peak, the chemokine CXC motif ligand 14 (CXCL14) gene, which is thought to be a potent chemoattractant and activator of dendritic cells. ${ }^{28}$

\section{Analysis of loci reported in previous GWAS of asthma}

Despite low power to detect risk loci for asthma with genome-wide significance, our whole-genome data set nonetheless provided an opportunity to attempt to replicate previously identified loci, given 
Table 2 SNP association results for ORMDL3, PDE4D, DENND1B and IL1RL1

\begin{tabular}{|c|c|c|c|c|c|c|c|c|c|}
\hline \multirow[b]{2}{*}{ Region } & \multirow[b]{2}{*}{ Gene } & \multirow[b]{2}{*}{ Literature $S N P$, allele } & \multirow[b]{2}{*}{ Reported OR } & \multirow[b]{2}{*}{ Power, \% ${ }^{\mathrm{b}}$} & \multicolumn{2}{|c|}{ Allele frequency } & \multirow[b]{2}{*}{ OR } & \multirow[b]{2}{*}{$95 \% \mathrm{Cl}$ OR } & \multirow[b]{2}{*}{ P-value } \\
\hline & & & & & Cases & Controls & & & \\
\hline \multicolumn{10}{|c|}{ (a) Most-associated variant reported in the original studies } \\
\hline $17 q 12-21$ & ORMDL3 & rs7216389, T & 1.45 & 100 & 0.53 & 0.47 & 1.25 & $1.12-1.40$ & $6.0 \times 10^{-5}$ \\
\hline $5 q 12$ & $P D E 4 D$ & rs1588265, G & 0.85 & 78 & 0.31 & 0.30 & 1.05 & $0.93-1.18$ & 0.4334 \\
\hline $1 q 31$ & DENND1B & rs1775456, G & 0.75 & 93 & 0.21 & 0.20 & 1.02 & $0.89-1.17$ & 0.7862 \\
\hline \multirow[t]{2}{*}{$2 q 12$} & ILIRLI & rs1420101, T & 1.16 & 77 & 0.39 & 0.38 & 1.04 & $0.93-1.17$ & 0.4649 \\
\hline & & & & & \multicolumn{2}{|c|}{ Allele frequency } & & \multicolumn{2}{|c|}{$\mathrm{P}$-value } \\
\hline Region & Gene & Best SNP, allele & N SNPs tested ${ }^{\mathrm{c}}$ & $\angle D$ with literature $S N P, \mathrm{r}^{\mathrm{b}}$ & Cases & Controls & $O R$ & Uncorrected & Corrected $^{\mathrm{d}}$ \\
\hline \multicolumn{10}{|c|}{ (b) Most-associated variant in this study } \\
\hline $17 q 12-21$ & ORMDL3 & rs6503525,C & 232 & 0.61 & 0.50 & 0.43 & 1.33 & $4.8 \times 10^{-7}$ & 0.0002 \\
\hline $5 q 12$ & $P D E 4 D$ & rs17725522,C & 1503 & 0.00 & 0.06 & 0.08 & 0.68 & 0.0007 & 0.1948 \\
\hline 1q31 & DENND1B & rs12094462,G & 171 & 0.03 & 0.10 & 0.12 & 0.81 & 0.0229 & 0.3238 \\
\hline $2 q 12$ & ILIRLI & rs10197862,G & 182 & 0.09 & 0.12 & 0.16 & 0.75 & 0.0004 & 0.0122 \\
\hline
\end{tabular}

${ }^{a}$ All four SNPs in (a) were present in the Illumina chips used and passed QC filters. The top SNPs listed in (b) were imputed with high confidence with MACH, with nearby genotyped SNPS

providing comparable evidence for association (cf. Figure 1).

bower to detect the initial association at $\alpha=0.05$ in this study.

'Number of SNPs located in or within $50 \mathrm{~kb}$ of each gene. For ORMDL3, we considered all SNPs located between $50 \mathrm{~kb}$ downstream of GSDMB and $50 \mathrm{~kb}$ upstream of GSDMA.

dSignificance of the best SNP after accounting for the number of and LD between all SNPs tested in the respective gene, estimated from 10000 permutations.

the less stringent threshold required for significance. We, therefore, investigated whether there was any evidence for association with SNPs located in the three remaining loci that have previously been reported to have reproducible effects on asthma: PDE4D, DENND1B and $I L 1 R L 1$. For completeness, we also report results from these analyses for ORMDL3.

We first focused on the individual variant with strongest association reported in the original study for each locus. We found consistent evidence for association with the leading literature SNP for ORMDL3 (rs7216389, $\mathrm{OR}=1.25, P=6 \times 10^{-5}$ ), but not for the three remaining loci, despite reasonable power (Table $2 \mathrm{a}$ ).

To address the possibility that allelic heterogeneity could explain the negative results for PDE4D, DENND1B and IL1RL1, we then extended the analysis to all variants available in our study for each of these loci $( \pm 50 \mathrm{~kb})$. A variant in IL1RL1 was found to be associated with asthma after accounting for the number of and LD between SNPs in the gene (Table 2b); in contrast, there was no overall evidence for association with PDE4D or DENND1B (Table 2b and Figure 1).

The peak variant for IL1RL1 was rs10197862 (OR=0.75 for the G allele, $P=0.0004$, corrected $P=0.01)$, which is in low $\operatorname{LD}\left(r^{2}=0.09\right)$ with the rs1420101 variant reported by Gudbjartsson et al. ${ }^{16}$ Haplotype analysis of both variants indicates that the rs10197862:G protective allele is found exclusively on a haplotype that also carries the rs1420101:C protective allele (not shown). Although this GC haplotype could be a proxy for a single functional variant that explains both associations, this is unlikely given that with a high-risk allele frequency of $84 \%$, the associated effect size for such variant would have to be high (OR of $\sim 1.8$ ) to match the results reported for rs1420101 $(\mathrm{OR}=1.15)$ by Gudbjartsson et al. ${ }^{16}$ As rs10197862 was also in low LD $\left(r^{2}<0.1\right)$ with two nearby variants (rs2310173 and rs917997) reported recently to associate with other immune-related diseases, ${ }^{29,30}$ our results suggest that rs10197862 represents or tags an independent functional variant in the $2 \mathrm{q} 12$ region with significant effects on asthma risk.

Given that the IL1RL1 and ORMDL3 loci have also been reported to associate with celiac ${ }^{29}$ and Crohn's disease, ${ }^{31}$ respectively, we investigated whether there was any evidence for association between asthma and other confirmed loci for these diseases. Among these, no single locus was associated with asthma risk after accounting for the number of SNPs considered (Supplementary Tables 3 and 4).

We extended our genome-wide search for asthma risk loci by considering $\mathrm{CNV}$ data that were obtained for 759 adolescents, including 270 doctor-diagnosed asthma cases and 489 controls.

Given that common CNVs were likely tagged by SNPs tested in the genome-wide analysis, ${ }^{18}$ we focused on large $(>100 \mathrm{~kb}$ and $<1 \mathrm{Mb}$ ), uncommon (MAF <0.05) deletions or duplications identified with high confidence. A total of 2681 such segments were detected, with a median of three CNVs per individual (mean $=3.9$, range $1-21$ ); the median CNV size was $198 \mathrm{~kb}$ (mean $252 \mathrm{~kb}$, range $100-998 \mathrm{~kb}$ ). Of these, 1621 (61\%) were deletions and 1060 were duplications (39\%). These CNVs mapped to 244 (deletions) and 230 (duplications) nonoverlapping regions of the genome, with a median region length of $259 \mathrm{~kb}$ (range $102 \mathrm{~kb}-4.0 \mathrm{Mb}$ ) and $281 \mathrm{~kb}$ (range $102 \mathrm{~kb}-3.6 \mathrm{Mb}$ ), respectively.

We performed two sets of CNV association analyses. First, we tested whether the frequency of deletions or duplications at individual loci were associated with asthma status. After accounting for all the regions tested, no single locus was significantly associated with asthma; the two most-associated regions are shown in Table 3, including a $102-775 \mathrm{~kb}$ deletion of chromosome 1q21 and a $129-432 \mathrm{~kb}$ deletion of chromosome $17 \mathrm{q} 21$. To visually validate the $\mathrm{CNV}$ calls made by QuantiSNPv1.1 for these two regions, we inspected the local log $\mathrm{R}$ ratio values for the individual samples identified by the software as carrying a CNV. Log $\mathrm{R}$ ratio patterns for the $1 \mathrm{q} 21$ region were ambiguous (Supplementary Figure 3 ) and so this region may represent a technical artefact. In contrast, the $\log \mathrm{R}$ ratio patterns for all 38 individuals identified as carrying the chromosome $17 \mathrm{q} 21$ deletion are indeed consistent with a CNV in this region (Supplementary Figure 4), which is known to contain a common inversion in populations of European ancestry. ${ }^{32}$ The telomeric break point for this deletion was located in exon 13 of the NSF gene for 36 of the 38 individuals that carried this deletion, all of which belonged to the $\mathrm{H} 2$ haplotype as defined by the inversion-informative rs 1800547 variant. $^{32}$

Second, we tested the association between genome-wide CNV burden and asthma risk. We hypothesised that the cumulative effect of multiple deletions or duplications across an individual's genome 
could significantly increase the risk of developing asthma. After correcting for the number of hypotheses tested, we found no evidence that cases and controls had significant differences in the total number of CNVs, length of CNVs or in the number of genes affected by a $\mathrm{CNV}$, either when considering deletions or duplications (Table 4).

\section{Follow-up of top SNP and CNV associations}

Finally, we attempted to replicate the most-associated loci identified in the whole-genome SNP and CNV analyses, as well as the novel IL1RL1 association, in an independent cohort of 391 doctor-diagnosed asthmatics and 213 controls (Supplementary Table 5). Power to nominally replicate these associations was relatively modest (30-60\%), but we observed a convincing association between the IL1RL1 variant rs10197862 and asthma $\left(\mathrm{OR}=0.58, P=1.2 \times 10^{-5}\right)$, which confirms the presence of a novel independent risk allele in this region (Supplementary Table 6). Also consistent with our initial observation, the 17 q21 deletion was more frequent in asthma cases (5/391) than controls (1/213) (Supplementary Figure 5), but these effects were not significant (Supplementary Table 6). The different frequency estimates for this deletion between the discovery (5\%) and replication (1\%) panels suggests that its prevalence is underestimated in the latter (as all deletions were visually validated) and highlights the technical

Table 3 Individual regions with strongest association between CNVs and asthma risk

\begin{tabular}{lcc}
\hline Chromosome & $1 \mathrm{q} 21$ & $17 \mathrm{q} 21$ \\
CNV type & Deletion & Deletion \\
Average CNV span & $283(102-775)$ & $320(129-432)$ \\
(range) $(\mathrm{kb})$ & & \\
Range shared region (bp) & $146397003-146438291$ & $42008822-42137359$ \\
Length shared region (kb) & 41 & 129 \\
$N$ cases with CNV/total & $8 / 270$ & $20 / 270$ \\
$N$ controls with CNV/total & $2 / 489$ & $18 / 489$ \\
OR & 7 & 2 \\
$P$-value & 0.0051 & 0.0196 \\
Corrected $P$-value & 0.4403 & 0.9947 \\
Genes in region $\pm 50 \mathrm{~kb}$ & NBPF1, NBPF10, NBPF12, & ARL17, ARL17P1, \\
& NBPF14, PPIAL4A & ARL17A, ARL17B, NSF \\
SNP most correlated with & rs7554500 (0.05) & rs7225002 (0.30) \\
CNV $\left({ }^{2}\right.$ ) & & \\
Visual validation of CNVs ${ }^{\text {b }}$ & Ambiguous & Yes \\
\hline
\end{tabular}

asignificance after correcting for all deletions or duplications tested across the genome, estimated from 10000 permutations.

bLocal $\log \mathrm{R}$ ratio patterns for individuals identified as carrying a deletion were inspected to validate the respective CNV calls produced by QuantiSNPv1.1. Intensity patterns for the validate the respective $\mathrm{CNV}$ calls produced by QuantiSNPv1.1. Intensity patter $17 q 21$ but not for the $1 \mathrm{q} 21$ region
(Supplementary Figures 3 and 4 ). limitations of CNV detection using genotyping arrays, even when the same array (Illumina 610K) and CNV calling pipeline are used across projects. Further analyses in larger cohorts are thus warranted to confirm this new putative association.

\section{DISCUSSION}

Our analysis of whole-genome SNP data in 986 self-reported asthma cases and 1846 controls confirms that variants in ORMDL3, a locus previously reported to reproducibly associate with asthma in European and North American populations, ${ }^{7}$ influences disease risk in the Australian population. The most-associated variant in our analysis was rs6503525, which lies $39 \mathrm{~kb}$ upstream of ORMDL3. Although gene expression results point to ORMDL3 as the most likely causal candidate at $17 \mathrm{q} 12$, we note that other nearby genes should not be neglected in future experimental work conducted under this broad region of high LD. Specifically, on a recent analysis of genetic determinants of peripheral blood lymphocyte cell levels, ${ }^{33}$ we found that a synonymous change (rs907092) in the Ikaros Family Zinc Finger 3 (IKZF3), which is located $57 \mathrm{~kb}$ downstream of ORMDL3, was a significant predictor of B cell levels in adolescents. The $G$ allele that associated with increased numbers of $\mathrm{B}$ cells in that analysis had a predisposing effect for asthma in this study $\left(\mathrm{OR}=1.22, P=5.1 \times 10^{-4}\right)$. This variant is in partial LD with rs6503525 $\left(r^{2}=0.52\right)$ and, although it may simply tag the same underlying causal variant, it may also represent an additional risk locus in this region. Further studies that explore this possibility are warranted.

Our results also demonstrate that genetic variation in IL1RL1 significantly influences asthma risk, but the specific variant identified in our study (rs10197862) is independent of that reported previously to influence eosinophil levels and asthma by Gudbjartsson et al. ${ }^{16}$ These results thus indicate that multiple independent variants in IL1RL1 can contribute to asthma risk and further emphasise the need to apply gene-based association tests that can effectively account for such effects. Two additional variants near the neighbouring interleukin genes IL18R1 and IL1R2 have recently been reported to associate with celiac disease ${ }^{29}$ and ankylosing spondylitis, ${ }^{30}$ but these were in low LD with rs10197862. Together, these data indicate that the $2 \mathrm{q} 12$ region has an important and diverse function in the regulation of immune responses.

PDE4D and DENND1B were recently identified as asthma susceptibility genes. ${ }^{9,11}$ Our analyses do not support these findings, either when focusing on the individual variants reported by the original studies or after extending to all the genetic variation tested in each gene in our study. Failure to replicate the reported associations with PDE4D and DENND1B could represent a false-negative finding in our study (eg limited power to detect an actual effect size that is smaller than that estimated in the original studies), but could also indicate

Table 4 Association results between genome-wide CNV burden and asthma risk in 270 asthma cases and 489 controls

\begin{tabular}{|c|c|c|c|c|c|c|c|c|}
\hline & \multicolumn{4}{|c|}{ Deletions } & \multicolumn{4}{|c|}{ Duplications } \\
\hline & \multirow[t]{2}{*}{ Cases } & \multirow[t]{2}{*}{ Controls } & \multicolumn{2}{|c|}{ P-value ${ }^{a}$} & \multirow[t]{2}{*}{ Cases } & \multirow[t]{2}{*}{ Controls } & \multicolumn{2}{|c|}{ P-value ${ }^{a}$} \\
\hline & & & Uncorrected & Corrected & & & Uncorrected & Corrected \\
\hline$N$ CNVs detected & 564 & 1057 & - & - & 326 & 734 & - & - \\
\hline NCNVs/individual & 2.09 & 2.16 & 0.8305 & 0.9989 & 1.21 & 1.50 & 0.0658 & 0.2352 \\
\hline Total CNV span (kb) & 693.5 & 695.2 & 0.9371 & 1.0000 & 476.7 & 572.2 & 0.0282 & 0.0994 \\
\hline Average CNV span (kb) & 223.3 & 237.3 & 0.4115 & 0.8181 & 259.1 & 269.1 & 0.5835 & 0.9623 \\
\hline$N$ genes spanned by CNVs & 6.67 & 7.31 & 0.4318 & 0.8165 & 4.49 & 4.01 & 0.0675 & 0.2180 \\
\hline
\end{tabular}

aTwo-sided $P$-value estimated from 10000 permutations. Corrected $P$-values were obtained after adjusting for the overall number of hypotheses tested ( $N=8$ ) through the analysis of the permuted data sets. 
that these genes represent false-positive discoveries. Further analyses in large cohorts are thus warranted to confirm both genes as true asthma susceptibility loci.

We also show that CNVs with strong effects on asthma could not be detected with experiment-wide significance in a subset of 270 cases and 489 controls. However, it is noteworthy that the chromosome $17 \mathrm{q} 21$ deletion that associated with increase asthma risk in our analysis overlaps with the known $900 \mathrm{~kb}$ inversion ${ }^{32}$ that was recently reported to associate with corticosteroid response in asthma. ${ }^{34} \mathrm{We}$ thus provide some further support for the association between structural variants in chromosome $17 \mathrm{q} 21$ and asthma, but further studies are required to carefully characterise this complex region of the genome. Last, results from our genome-wide CNV burden analysis suggest that asthma cases do not have increased numbers of uncommon large chromosomal deletions or duplications when compared with unaffected individuals. The number of genes affected by CNVs was also comparable between both groups. These results contrast with recent reports for psychiatric diseases, namely schizophrenia. ${ }^{21}$ In our analysis, we observed significant confounding effects of technical variables, such as array batch effects. Ignoring these may result in false-positive results, particularly when cases and controls have been genotyped in different batches or at different times.

There are a number of limitations that should be considered when interpreting our results. First, our definition of lifetime asthma status is somewhat loose, as it was based on self-reported information provided in surveys that often included comparable but not identical questions, and did not exclude co-morbid diseases such as COPD. However, we note that despite these differences, the resulting phenotype is heritable $(61 \%)$ and allowed us to unambiguously confirm the association with ORMDL3, effectively a positive control for asthma. Second, the sample size analysed was small for a GWAS of a complex disease. These results will now be combined with other ongoing studies to provide a more powerful and comprehensive survey of asthma risk factors. Third, age-at-onset information was only available for a subset of cases and so it was not considered in our analysis. Nonetheless, we note that to date the only locus with confirmed age-at-onset effects is ORMDL3, a locus that we replicate in this study. Last, our analysis of rare structural variants relied heavily on the ability to identify such segments from SNP and CNV probe intensity patterns, an approach that can be influenced by a number of technical artefacts; the sample size used was also very modest. Thus, confirmation of our results by independent studies are warranted, particularly the putative association with the $17 \mathrm{q} 21$ deletion.

In conclusion, we report a convincing association between asthma risk and both novel and previously reported sequence variants in two loci, IL1RL1 and ORMDL1; we also identify a $300 \mathrm{~kb}$ deletion on chromosome 17q21 with putative effects on disease risk. We found no evidence for association with $P D E 4 D$ or DENND1B, and no indication that asthmatics have more or larger CNVs than non-asthmatics. These results may help direct future studies of asthma genetics.

\section{CONFLICT OF INTEREST}

The authors declare no conflict of interest.

\section{ACKNOWLEDGEMENTS}

We thank the twins and their families for their participation; Dixie Statham, Ann Eldridge, Marlene Grace and Kerrie McAloney for sample collection; Lisa Bowdler and Steven Crooks for DNA processing; David Smyth, Harry Beeby and Daniel Park for IT support. Funding was provided by the Australian National Health and Medical Research Council (241944, 339462, 389927, 389875, 389891, 389892, 389938, 442915, 442981, 496739, 552485, 552498,
613627), the Australian Research Council (A7960034, A79906588, A79801419, DP0770096, DP0212016, DP0343921), the FP-5 GenomEUtwin Project (QLG2-CT-2002-01254) and the US National Institutes of Health (NIH Grants AA07728, AA07535, AA10248, AA11998, AA13320, AA13321, AA13326, AA14041, DA12854, MH66206). A portion of the genotyping on which this study was based (Illumina 370k scans on 4300 individuals) was carried out at the Center for Inherited Disease Research, Baltimore (CIDR) through an access award to our late colleague Dr Richard Todd (Psychiatry, Washington University School of Medicine, St Louis). SEM, DRN, MARF, GWM and PMV are supported by the NHMRC Fellowship Scheme.

1 Australian Centre for Asthma Monitoring. Asthma in Australia 2008. AlHW Asthma Series No. 3. Cat. No. ACM 14. Canberra: AIHW, 2008.

2 Lemanske Jr RF, Busse WW: Asthma: clinical expression and molecular mechanisms. J Allergy Clin Immunol 2010; 125: S95-102.

3 Altshuler D, Daly MJ, Lander ES: Genetic mapping in human disease. Science 2008; 322: 881-888.

4 Hindorff LA, Sethupathy P, Junkins HA et al: Potential etiologic and functional implications of genome-wide association loci for human diseases and traits. Proc Natl Acad Sci USA 2009; 106: 9362-9367.

5 Rioux JD, Xavier RJ, Taylor KD et al: Genome-wide association study identifies new susceptibility loci for Crohn disease and implicates autophagy in disease pathogenesis. Nat Genet 2007; 39: 596-604.

6 Fellay J, Shianna KV, Ge D et al: A whole-genome association study of major determinants for host control of HIV-1. Science 2007; 317: 944-947.

7 Moffatt MF, Kabesch M, Liang L et al: Genetic variants regulating ORMDL3 expression contribute to the risk of childhood asthma. Nature 2007; 448: 470-473.

8 Choudhry S, Taub M, Mei R et al: Genome-wide screen for asthma in Puerto Ricans: evidence for association with 5q23 region. Hum Genet 2008; 123: 455-468.

9 Himes BE, Hunninghake GM, Baurley JW et al: Genome-wide association analysis identifies PDE4D as an asthma-susceptibility gene. Am J Hum Genet 2009; 84: 581-593.

10 Hancock DB, Romieu I, Shi M et al: Genome-wide association study implicates chromosome 9q21.31 as a susceptibility locus for asthma in Mexican children. PLoS Genet 2009; 5: e1000623.

11 Sleiman PM, Flory J, Imielinski M et al: Variants of DENND1B associated with asthma in children. N Engl J Med 2010; 362: 36-44.

12 Mathias RA, Grant AV, Rafaels N et al: A genome-wide association study on African-ancestry populations for asthma. J Allergy Clin Immunol 2010; 125: 336-346.e4.

$13 \mathrm{Li} \mathrm{X,} \mathrm{Howard} \mathrm{TD,} \mathrm{Zheng} \mathrm{SL} \mathrm{et} \mathrm{al:} \mathrm{Genome-wide} \mathrm{association} \mathrm{study} \mathrm{of} \mathrm{asthma}$ identifies RAD50-IL13 and HLA-DR/DQ regions. J Allergy Clin Immunol 2010; 125: 328-335.e11.

14 Ober C, Tan Z, Sun Y et al: Effect of variation in CHI3L1 on serum YKL-40 level, risk of asthma, and lung function. N Engl J Med 2008; 358: 1682-1691.

15 Weidinger S, Gieger C, Rodriguez E et al: Genome-wide scan on total serum IgE levels identifies FCER1A as novel susceptibility locus. PLoS Genet 2008; 4: e1000166.

16 Gudbjartsson DF, Bjornsdottir US, Halapi E et al: Sequence variants affecting eosinophil numbers associate with asthma and myocardial infarction. Nat Genet 2009; 41: 342-347.

17 Scherer SW, Lee C, Birney E et al: Challenges and standards in integrating surveys of structural variation. Nat Genet 2007; 39: S7-15.

18 The Wellcome Trust Case Control Consortium: Genome-wide association study of CNVs in 16000 cases of eight common diseases and 3000 shared controls. Nature 2010; 464: 713-720.

19 Bochukova EG, Huang N, Keogh J et al: Large, rare chromosomal deletions associated with severe early-onset obesity. Nature 2010; 463: 666-670.

20 Weiss LA, Shen Y, Korn JM et al: Association between microdeletion and microduplication at 16p11.2 and autism. N Engl J Med 2008; 358: 667-675.

21 The International Schizophrenia Consortium: Rare chromosomal deletions and duplications increase risk of schizophrenia. Nature 2008; 455: 237-241.

22 Ionita-Laza I, Perry GH, Raby BA et al: On the analysis of copy-number variations in genome-wide association studies: a translation of the family-based association test. Genet Epidemiol 2008; 32: 273-284.

23 Medland SE, Nyholt DR, Painter JN et al: Common variants in the trichohyalin gene are associated with straight hair in Europeans. Am J Hum Genet 2009; 85: 750-755.

24 Blangero J, Almasy L: Multipoint oligogenic linkage analysis of quantitative traits. Genet Epidemiol 1997; 14: 959-964.

25 Purcell S, Neale B, Todd-Brown K et al: PLINK: a tool set for whole-genome association and population-based linkage analyses. Am J Hum Genet 2007; 81: 559-575.

26 Frazer KA, Ballinger DG, Cox DR et al: A second generation human haplotype map of over 3.1 million SNPs. Nature 2007; 449: 851-861.

27 Colella S, Yau C, Taylor JM et al: QuantiSNP: an Objective Bayes Hidden-Markov Mode to detect and accurately map copy number variation using SNP genotyping data. Nucleic Acids Res 2007; 35: 2013-2025. 
28 Shurin GV, Ferris RL, Tourkova IL et al: Loss of new chemokine CXCL14 in tumor tissue is associated with low infiltration by dendritic cells (DC), while restoration of human CXCL14 expression in tumor cells causes attraction of DC both in vitro and in vivo. $\mathrm{J}$ Immunol 2005; 174: 5490-5498.

29 Dubois PC, Trynka G, Franke L et al: Multiple common variants for celiac disease influencing immune gene expression. Nat Genet 2010; 42: 295-302.

30 Australo-Anglo-American Spondyloarthritis Consortium (TASC)Reveille JD, Sims AM, Danoy $\mathrm{P}$ et al: Genome-wide association study of ankylosing spondylitis identifies non-MHC susceptibility loci. Nat Genet 2010; 42: 123-127.
31 Barrett JC, Hansoul S, Nicolae DL et al: Genome-wide association defines more than 30 distinct susceptibility loci for Crohn's disease. Nat Genet 2008; 40: 955-962.

32 Stefansson $\mathrm{H}$, Helgason A, Thorleifsson $\mathrm{G}$ et al: A common inversion under selection in Europeans. Nat Genet 2005; 37: 129-137.

33 Ferreira MA, Mangino M, Brumme CJ et al: Quantitative trait loci for CD4: CD8 lymphocyte ratio are associated with risk of type 1 diabetes and HIV-1 immune control. Am J Hum Genet 2010; 86: 88-92.

34 Tantisira KG, Lazarus R, Litonjua AA, Klanderman B, Weiss ST: Chromosome 17: association of a large inversion polymorphism with corticosteroid response in asthma. Pharmacogenet Genomics 2008; 18: 733-737.

Supplementary Information accompanies the paper on European Journal of Human Genetics website (http://www.nature.com/ejhg) 